\title{
Modelagem de funções no cálculo dos índices DRIS
}

\author{
Paulo Guilherme Salvador Wadt ${ }^{(1)}$, Davi José Silva ${ }^{(2)}$, Celsemy Eleuterio Maia( ${ }^{(3)}$, Juarez Barbosa Tomé Júnior ${ }^{(4)}$, \\ Paulo Augusto da Costa Pinto(5) e Pedro Luiz Oliveira de Almeida Machado(6)
}

\begin{abstract}
(1)Embrapa Acre, Caixa Postal 321, CEP 69908-970 Rio Branco, AC. E-mail: paulo@cpafac.embrapa.br (2)Embrapa Semi-Árido, Caixa Postal 23, CEP 56302-970 Petrolina, PE. E-mail: davi@cpatsa.embrapa.br (3)Universidade Federal Rural do Semi-Árido, Av. Professor Antonio Campos, no 10, Costa e Silva, CEP 59625-620 Mossoró, RN. E-mail: celsemy@ufersa.edu.br (4)Embrapa Sede, Parque Estação Biológica s/no, Edifício Sede, CEP 70770-901 Brasília, DF. E-mail: juarez.tome@embrapa.br (5)Universidade Estadual da Bahia, Dep. de Tecnologia e Ciências Sociais, Av. Edgard Chastinet s/no, CEP 48900-000 Juazeiro, BA. E-mail: pacostapinto@yahoo.com.br (6)Embrapa Arroz e Feijão, Caixa Postal 179, CEP 75375-000 Santo Antônio de Goiás, GO. E-mail: pmachado@cnpaf.embrapa.br
\end{abstract}

Resumo - $\mathrm{O}$ objetivo deste trabalho foi modelar o fator $\mathrm{k}$ e as funções DRIS para a diagnose foliar de mangueiras cultivadas. Dez pomares comerciais, no estágio de produção, localizados no vale do Rio São Francisco, foram monitorados, mensalmente, durante dois anos, por meio da coleta de amostras foliares para determinação dos teores de N, P, K, Ca, Mg, B, Fe, Mn, Zn e Cu. Os dados foram testados quanto à normalidade e as relações entre as concentrações dos nutrientes foram usadas para calcular as normas DRIS, obtendo-se média, variância e limites máximo e mínimo de cada relação dentro da população amostrada. Os nutrientes foram classificados como macronutrientes de resposta freqüente $(\mathrm{MAF})(\mathrm{N}, \mathrm{Pe} \mathrm{K})$, macronutrientes de resposta rara (MAR) $(\mathrm{Ca}$ e $\mathrm{Mg}$ ); micronutrientes de resposta freqüente (MIF) (B, Fe, Mn e Zn) e, micronutrientes de resposta rara (MIR) $(\mathrm{Cu})$. Funções DRIS foram desenvolvidas para cada classe de nutrientes. O modelo desenvolvido expressa o balanço nutricional das plantas cultivadas ajustado a cada nutriente e reflete o comportamento biológico das plantas como resultado da variação da disponibilidade dos nutrientes.

Termos para indexação: Mangifera indica, normas DRIS, diagnose foliar.

\section{Modelling of functions in calculating DRIS indices}

\begin{abstract}
The objective of this work was to model DRIS functions and $\mathrm{k}$ factor on foliar diagnosis of mango crops. Ten commercial orchards, at yield stage, in the São Francisco River valley were monitored, monthly, during a two-year period, by means of collecting leaf samples to determine $\mathrm{N}, \mathrm{P}, \mathrm{K}, \mathrm{Ca}, \mathrm{Mg}, \mathrm{B}, \mathrm{Fe}, \mathrm{Mn}, \mathrm{Zn}$ and $\mathrm{Cu}$ concentrations. Data were tested for normality and bivariate relationships between nutrient concentrations were used to calculate DRIS norms. Mean, variance as well as minimum and maximum values were calculated for each relationship within the population. Nutrients were classified as follows: highly responsive macronutrients (HRMa) $(\mathrm{N}, \mathrm{P}$ and $\mathrm{K})$; rarely responsive macronutrients (RRMa) $(\mathrm{Ca}$ and $\mathrm{Mg})$; highly responsive micronutrients (HRMi) (B, Fe, Mn and $\mathrm{Zn}$ ), and rarely responsive micronutrients (RRMi) $(\mathrm{Cu})$. DRIS functions were developed for each nutrient class. Results showed that the developed model expresses the nutritional balance adjusted for each nutrient, and reflects the expected biological behavior of plants as a result of variation in the availability of nutrients.
\end{abstract}

Index terms: Mangifera indica, DRIS norms, foliar diagnosis.

\section{Introdução}

O sistema integrado de diagnose e recomendação (DRIS) preconiza a utilização do maior número possível de fatores relacionados à produtividade das culturas (Beaufils, 1973), embora, inicialmente, apenas três macronutrientes, N, P e K, fossem utilizados (Beaufils, 1973; Sumner, 1977a, 1977b).

Com a utilização de mais nutrientes para o diagnóstico nutricional, novas aproximações ou fórmulas DRIS passaram a ser recomendadas (Jones, 1981; Elwali \& Gascho, 1984; Hallmark et al., 1989; Parent et al., 1994), as quais, junto às propostas originais de Beaufils (1973) e Jones (1981), são atualmente utilizadas (Partelli et al., 2005; Silva et al., 2005; Silveira et al., 2005), até mesmo na cultura da mangueira (Shaffer et al., 1988; Pinto, 2002).

Sumner (1999) argumenta que a fórmula original de Beaufils (1973) foi desenvolvida para corrigir desvios de simetria entre as relações calculadas na forma direta 
e inversa das funções DRIS. A fórmula de Jones (1981), por sua vez, é considerada uma simplificação da fórmula original e seria baseada em princípios estatísticos mais rígidos (Alvarez Venegas \& Leite, 1992).

Contudo, Maia (1999) sugere que as alterações realizadas na fórmula original foram puramente arbitrárias, já que a fórmula original possibilita modelar o comportamento biológico da planta quanto às relações nutricionais e, portanto, transfere ao método DRIS maior possibilidade de se desenvolver funções DRIS ajustadas, conforme o tipo de nutriente e sua situação de deficiência ou excesso. Além disso, Maia (1999) destaca que o fator $\mathrm{k}$, denominado constante de sensibilidade (Bataglia \& Santos, 1990; Nick, 1998), consiste fundamentalmente em um fator de escala, caso seja adotado o mesmo valor para qualquer relação entre dois nutrientes. Por sua vez, Bataglia et al. (2004) indicam pequena melhoria no diagnóstico nutricional utilizando a variável $\mathrm{k}$ ajustada de acordo com o grau de resposta do nutriente à adubação em não responsivos, mediamente responsivos e responsivos.

O desenvolvimento do sistema DRIS tem sido realizado com base em critérios para separar nutrientes limitantes dos não limitantes (Hallmark et al., 1987; Wadt, 1996, 2004) e para identificar relações nutricionais úteis ao diagnóstico (Jones, 1981; Wadt, 1996; Wadt et al., 1999). Entretanto, o desenvolvimento de novas funções DRIS representa importante alternativa para modelar a resposta biológica das culturas às variações na disponibilidade de nutrientes.

O objetivo deste trabalho foi avaliar a modelagem das funções DRIS e do fator k como alternativa na avaliação nutricional de lavouras, tendo como base mangueiras cultivadas na região do Submédio São Francisco, no Semi-Árido nordestino.

\section{Material e Métodos}

Os dados de campo foram obtidos em lavouras de mangueiras (Mangifera indica L.) da variedade Tommy Atkins, cultivadas sob irrigação. Foram avaliados mensalmente, durante dois anos, dez pomares localizados em propriedades agrícolas do Submédio São Francisco, na região Semi-Árida do nordeste do Brasil. Essas propriedades estão situadas nos municípios de Petrolina, PE, Juazeiro e Casa Nova, BA. Os solos desses pomares apresentam características diversificadas, com fertilidade de baixa a alta, textura arenosa a argilosa, sendo classificados como Latossolos, Argissolos e Vertissolos. Em cada pomar foram selecionadas áreas de aproximadamente 5 ha, com idade superior a quatro anos e com árvores consideradas sadias. As avaliações foram realizadas durante 1998 e 1999.

A amostragem do material vegetal para análise foliar foi realizada mensalmente em plantas previamente marcadas. Foram coletadas folhas maduras, inteiras e sadias, com 4 a 7 meses de idade, em uma única posição na planta, preferencialmente na penúltima brotação, ou na brotação terminal. Foram coletadas quatro folhas por planta, nas posições referentes aos pontos cardeais, na altura mediana da planta, em 25 árvores por área, de acordo com Silva et al. (2002).

$\mathrm{O}$ material vegetal coletado foi acondicionado em sacos de papel e transportado para o laboratório, onde foram lavados, secados, moídos e submetidos às análises (Malavolta et al., 1997). Foram determinadas as concentrações de N, P, K, Ca e Mg (expressos em $\mathrm{g} \mathrm{kg}^{-1}$ ) e B, Fe, $\mathrm{Mn}, \mathrm{Zn}$ e $\mathrm{Cu}$ (expressos em $\mathrm{mg} \mathrm{kg}^{-1}$ ). Em seguida, os dados foram testados quanto à normalidade da variância e utilizados para o cálculo de normas DRIS preliminares. Para cada relação entre a concentração de dois nutrientes nas folhas, foram determinadas a média, a variância, e os valores máximos e mínimos das relações na população. Devido ao pequeno número de pomares amostrados, estes não foram subdivididos de acordo com a produtividade, já que consistiam de populações de plantas sadias (Beaufils, 1973).

Na modelagem das funções DRIS, os nutrientes foram separados em quatro grupos conforme princípios sugeridos por Baldock \& Schulte (1996) (grupo de macronutrientes de resposta freqüente (MAF): N, P e K; grupo de macronutrientes de resposta rara (MAR): $\mathrm{Ca}$ e $\mathrm{Mg}$; grupo de micronutrientes de resposta freqüente (MIF): B, Fe, $\mathrm{Mn}$ e $\mathrm{Zn}$ e grupo de micronutrientes de resposta rara (MIR): $\mathrm{Cu}$. Em cada grupamento, foram definidas as funções DRIS.

No grupo MAF, a função DRIS foi alterada para acrescentar o fator de correção à formula de Jones (1981), a fim de superestimar a deficiência e subestimar o excesso.

$\mathrm{Se} \mathrm{A} / \mathrm{B}<\mathrm{a} / \mathrm{b}$, a função equivale à fórmula original de Beaufils, superestimando a deficiência nutricional (Maia, 1999). Se A/B>a/b, a mesma função apresentará uma nova curvatura, subestimando o excesso nutricional: 
$\mathrm{f}(\mathrm{A} / \mathrm{B})=\left(\frac{\mathrm{A} / \mathrm{B}-\mathrm{a} / \mathrm{b}}{\mathrm{S}_{(\mathrm{a} / \mathrm{b})}}\right) \times \mathrm{k} \times\left(\frac{\mathrm{a} / \mathrm{b}}{\mathrm{A} / \mathrm{B}}\right)$.

No grupo MAR, a função DRIS foi alterada para incluir a correção a fim de subestimar tanto a deficiência como o excesso:

se $A / B<a / b: f(A / B)=\left(\frac{A / B-a / b}{S(a b)}\right) \times k \times\left(\frac{A / B}{a / b}\right) ;$

se $A / B>a / b: f(A / B)=\left(\frac{A / B-a / b}{S(a / b)}\right) \times k \times\left(\frac{a / b}{A / B}\right)$.

No grupo MIF, a função DRIS foi alterada para incluir a correção de modo a superestimar a deficiência e o excesso, além de considerar a possibilidade de efeito tóxico do nutriente: se $A / B<a / b: f(A / B)=\left(\frac{A / B-a / b}{S_{(a b)}}\right) \times k \times\left(\frac{a / b}{A / B}\right)$;

se $A / B>a / b$ e para nutriente com potencial de efeito tóxico quando em excesso:

$\mathrm{f}(\mathrm{A} / \mathrm{B})=\left(\frac{\mathrm{A} / \mathrm{B}-\mathrm{a} / \mathrm{b}}{\mathrm{S}_{(\mathrm{a} / \mathrm{b})}}\right) \times \mathrm{k} \times\left(\frac{\mathrm{A} / \mathrm{B}}{\mathrm{a} / \mathrm{b}}\right) ;$

se $A / B>a / b$ e para nutriente sem potencial de efeito tóxico quando em excesso:

$\mathrm{f}(\mathrm{A} / \mathrm{B})=\left(\frac{\mathrm{A} / \mathrm{B}-\mathrm{a} / \mathrm{b}}{\mathrm{S}_{(\mathrm{a} b)}}\right) \times \mathrm{k} \times 1$.

No grupo MIR, a função DRIS foi corrigida de modo a superestimar apenas o excesso:

se $A / B<a / b: f(A / B)=\left(\frac{A / B-a / b}{S_{(a / b)}}\right) \times k \times 1$;

se $A / B>a / b: f(A / B)=\left(\frac{A / B-a / b}{S_{(a b)}}\right) \times k \times\left(\frac{A / B}{a / b}\right)$.

A curvatura dessas funções foi ajustada pela variável $\mathrm{k}$ (fator de sensibilidade), com valores de 0,5, 1,0,1,5e 2,0.

As normas DRIS, preliminares, foram comparadas com os valores publicados por Pinto (2002) pelo teste t, a $1 \%$ de probabilidade, e o comportamento das funções DRIS foram avaliados graficamente em comparação com a fórmula de Jones (1981), por gráficos de dispersão, em que as funções DRIS foram as variáveis dependentes e as relações as variáveis independentes.

\section{Resultados e Discussão}

As normas preliminares utilizadas para o cálculo da função DRIS são apresentadas na Tabela 1. No total de 90 relações bivariadas, $76,7 \%$ dos valores médios de cada relação foram estatisticamente iguais, pelo teste $\mathrm{t}$ a $1 \%$ de probabilidade, aos valores publicados por Pinto (2002) para mangueiras Tommy Atkins do vale do São Francisco, enquanto outros $20 \%$ das relações foram

Tabela 1. Normas DRIS (média, variância, máximo, mínimo e número de observações para relações bivariadas) para mangueiras cultivadas na região do vale do Rio São Francisco, nos estados da Bahia e Pernambuco, comparadas com as normas obtidas por Pinto (2002).

\begin{tabular}{|c|c|c|c|c|c|c|}
\hline Relação & Média & Variância & Máximo & Mínimo & $\mathrm{n}$ & $\alpha^{(1)}$ \\
\hline $\mathrm{N} / \mathrm{P}$ & 14,09 & 15,39 & 27,91 & 6,00 & 145 & ig \\
\hline $\mathrm{N} / \mathrm{K}$ & 1,88 & 0,23 & 3,81 & 0,72 & 145 & ig \\
\hline $\mathrm{N} / \mathrm{Ca}$ & 0,88 & 0,15 & 3,42 & 0,22 & 145 & $\mathrm{ma}$ \\
\hline $\mathrm{N} / \mathrm{Mg}$ & 6,40 & 3,40 & 13,25 & 3,18 & 145 & ig \\
\hline $\mathrm{N} / \mathrm{Zn}$ & 0,55 & 0,22 & 4,20 & 0,04 & 145 & ig \\
\hline $\mathrm{N} / \mathrm{B}$ & 0,29 & 0,04 & 1,32 & 0,05 & 145 & $\mathrm{ma}$ \\
\hline $\mathrm{N} / \mathrm{Cu}$ & 0,56 & 0,21 & 2,03 & 0,03 & 145 & ig \\
\hline $\mathrm{N} / \mathrm{Fe}$ & 0,15 & 0,01 & 0,42 & 0,05 & 145 & ig \\
\hline N/Mn & 0,05 & 0,00 & 0,38 & 0,01 & 145 & $\mathrm{ma}$ \\
\hline $\mathrm{P} / \mathrm{N}$ & 0,08 & 0,00 & 0,17 & 0,04 & 145 & ig \\
\hline $\mathrm{P} / \mathrm{K}$ & 0,14 & 0,00 & 0,30 & 0,08 & 146 & ig \\
\hline $\mathrm{P} / \mathrm{Ca}$ & 0,07 & 0,00 & 0,39 & 0,02 & 146 & ig \\
\hline $\mathrm{P} / \mathrm{Mg}$ & 0,48 & 0,03 & 1,45 & 0,26 & 146 & ig \\
\hline $\mathrm{P} / \mathrm{Zn}$ & 0,04 & 0,00 & 0,28 & 0,00 & 146 & ig \\
\hline $\mathrm{P} / \mathrm{B}$ & 0,02 & 0,00 & 0,09 & 0,00 & 146 & ig \\
\hline $\mathrm{P} / \mathrm{Cu}$ & 0,04 & 0,00 & 0,14 & 0,00 & 146 & ig \\
\hline $\mathrm{P} / \mathrm{Fe}$ & 0,01 & 0,00 & 0,05 & 0,00 & 146 & ig \\
\hline $\mathrm{P} / \mathrm{Mn}$ & 0,00 & 0,00 & 0,03 & 0,00 & 146 & ig \\
\hline $\mathrm{K} / \mathrm{N}$ & 0,57 & 0,02 & 1,39 & 0,26 & 145 & ig \\
\hline $\mathrm{K} / \mathrm{P}$ & 7,62 & 2,75 & 13,30 & 3,32 & 146 & ig \\
\hline $\mathrm{K} / \mathrm{Ca}$ & 0,48 & 0,06 & 2,56 & 0,12 & 146 & ig \\
\hline $\mathrm{K} / \mathrm{Mg}$ & 3,56 & 1,49 & 13,50 & 1,86 & 146 & ig \\
\hline $\mathrm{K} / \mathrm{Zn}$ & 0,31 & 0,08 & 2,38 & 0,03 & 146 & ig \\
\hline $\mathrm{K} / \mathrm{B}$ & 0,15 & 0,01 & 0,68 & 0,03 & 146 & ig \\
\hline $\mathrm{K} / \mathrm{Cu}$ & 0,31 & 0,06 & 1,20 & 0,01 & 146 & ig \\
\hline $\mathrm{K} / \mathrm{Fe}$ & 0,08 & 0,00 & 0,31 & 0,02 & 146 & ig \\
\hline $\mathrm{K} / \mathrm{Mn}$ & 0,03 & 0,00 & 0,23 & 0,00 & 146 & $\mathrm{ma}$ \\
\hline $\mathrm{Ca} / \mathrm{N}$ & 1,32 & 0,29 & 4,50 & 0,29 & 145 & ig \\
\hline $\mathrm{Ca} / \mathrm{P}$ & 18,06 & 50,61 & 54,00 & 2,57 & 146 & ig \\
\hline $\mathrm{Ca} / \mathrm{K}$ & 2,41 & 0,88 & 8,31 & 0,39 & 146 & ig \\
\hline $\mathrm{Ca} / \mathrm{Mg}$ & 8,57 & 27,83 & 53,67 & 1,20 & 146 & ig \\
\hline $\mathrm{Ca} / \mathrm{Zn}$ & 0,79 & 1,56 & 10,80 & 0,05 & 146 & ig \\
\hline $\mathrm{Ca} / \mathrm{B}$ & 0,33 & 0,04 & 1,22 & 0,08 & 146 & $\mathrm{ma}$ \\
\hline $\mathrm{Ca} / \mathrm{Cu}$ & 0,73 & 0,56 & 5,40 & 0,03 & 146 & ig \\
\hline $\mathrm{Ca} / \mathrm{Fe}$ & 0,19 & 0,01 & 0,64 & 0,02 & 146 & ig \\
\hline $\mathrm{Ca} / \mathrm{Mn}$ & 0,07 & 0,01 & 0,41 & 0,01 & 146 & $\mathrm{ma}$ \\
\hline $\mathrm{Mg} / \mathrm{N}$ & 0,17 & 0,00 & 0,31 & 0,08 & 145 & ig \\
\hline $\mathrm{Mg} / \mathrm{P}$ & 2,28 & 0,39 & 3,85 & 0,69 & 146 & ig \\
\hline $\mathrm{Mg} / \mathrm{K}$ & 0,31 & 0,01 & 0,54 & 0,07 & 146 & ig \\
\hline $\mathrm{Mg} / \mathrm{Ca}$ & 0,14 & 0,01 & 0,83 & 0,02 & 146 & ig \\
\hline $\mathrm{Mg} / \mathrm{Zn}$ & 0,09 & 0,00 & 0,53 & 0,01 & 146 & ig \\
\hline $\mathrm{Mg} / \mathrm{B}$ & 0,05 & 0,00 & 0,23 & 0,00 & 146 & $\mathrm{ma}$ \\
\hline $\mathrm{Mg} / \mathrm{Cu}$ & 0,09 & 0,01 & 0,33 & 0,00 & 146 & ig \\
\hline $\mathrm{Mg} / \mathrm{Fe}$ & 0,03 & 0,00 & 0,10 & 0,00 & 146 & ig \\
\hline $\mathrm{Mg} / \mathrm{Mn}$ & 0,01 & 0,00 & 0,04 & 0,00 & 146 & $\mathrm{me}$ \\
\hline
\end{tabular}

Continua... 
Tabela 1. Continuação...

\begin{tabular}{|c|c|c|c|c|c|c|}
\hline Relação & Média & Variância & Máximo & Mínimo & $\mathrm{n}$ & $\alpha^{(1)}$ \\
\hline $\mathrm{Zn} / \mathrm{N}$ & 3,34 & 11,47 & 23,41 & 0,24 & 145 & $\mathrm{ma}$ \\
\hline $\mathrm{Zn} / \mathrm{P}$ & 45,99 & $2.039,63$ & 232,00 & 3,64 & 146 & $\mathrm{ma}$ \\
\hline $\mathrm{Zn} / \mathrm{K}$ & 6,22 & 40,50 & 34,12 & 0,42 & 146 & $\mathrm{ma}$ \\
\hline $\mathrm{Zn} / \mathrm{Ca}$ & 2,85 & 10,33 & 21,26 & 0,09 & 146 & $\mathrm{ma}$ \\
\hline $\mathrm{Zn} / \mathrm{Mg}$ & 20,26 & 361,93 & 111,67 & 1,90 & 146 & $\mathrm{ma}$ \\
\hline $\mathrm{Zn} / \mathrm{B}$ & 0,95 & 1,77 & 9,84 & 0,04 & 146 & $\mathrm{ma}$ \\
\hline $\mathrm{Zn} / \mathrm{Cu}$ & 1,49 & 3,14 & 12,52 & 0,05 & 146 & ig \\
\hline $\mathrm{Zn} / \mathrm{Fe}$ & 0,46 & 0,22 & 2,59 & 0,04 & 146 & ma \\
\hline $\mathrm{Zn} / \mathrm{Mn}$ & 0,12 & 0,02 & 0,69 & 0,01 & 146 & $\mathrm{ma}$ \\
\hline $\mathrm{B} / \mathrm{N}$ & 5,05 & 8,40 & 18,53 & 0,76 & 145 & $\mathrm{me}$ \\
\hline $\mathrm{B} / \mathrm{P}$ & 71,18 & $2.201,53$ & 325,93 & 10,77 & 146 & ig \\
\hline $\mathrm{B} / \mathrm{K}$ & 9,18 & 26,57 & 35,56 & 1,48 & 146 & me \\
\hline $\mathrm{B} / \mathrm{Ca}$ & 3,93 & 3,90 & 13,23 & 0,82 & 146 & ig \\
\hline $\mathrm{B} / \mathrm{Mg}$ & 34,26 & 891,99 & 253,33 & 4,36 & 146 & ig \\
\hline $\mathrm{B} / \mathrm{Zn}$ & 2,80 & 11,66 & 27,20 & 0,10 & 146 & ig \\
\hline $\mathrm{B} / \mathrm{Cu}$ & 3,00 & 12,51 & 23,47 & 0,04 & 146 & ig \\
\hline $\mathrm{B} / \mathrm{Fe}$ & 0,74 & 0,29 & 3,74 & 0,09 & 146 & ig \\
\hline $\mathrm{B} / \mathrm{Mn}$ & 0,28 & 0,18 & 3,14 & 0,01 & 146 & ig \\
\hline $\mathrm{Cu} / \mathrm{N}$ & 5,72 & 52,85 & 39,39 & 0,49 & 145 & ig \\
\hline $\mathrm{Cu} / \mathrm{P}$ & 85,47 & $15.740,31$ & 801,14 & 6,99 & 146 & ig \\
\hline $\mathrm{Cu} / \mathrm{K}$ & 11,35 & 256,43 & 90,75 & 0,83 & 146 & ig \\
\hline $\mathrm{Cu} / \mathrm{Ca}$ & 5,02 & 51,87 & 38,32 & 0,19 & 146 & ig \\
\hline $\mathrm{Cu} / \mathrm{Mg}$ & 35,15 & $2.009,01$ & 237,92 & 3,03 & 146 & ig \\
\hline $\mathrm{Cu} / \mathrm{Zn}$ & 2,44 & 11,24 & 19,27 & 0,08 & 146 & ig \\
\hline $\mathrm{Cu} / \mathrm{B}$ & 1,76 & 10,55 & 28,29 & 0,04 & 146 & ma \\
\hline $\mathrm{Cu} / \mathrm{Fe}$ & 0,78 & 1,02 & 5,11 & 0,05 & 146 & ig \\
\hline $\mathrm{Cu} / \mathrm{Mn}$ & 0,18 & 0,06 & 2,18 & 0,01 & 146 & ig \\
\hline $\mathrm{Fe} / \mathrm{N}$ & 8,13 & 14,36 & 19,46 & 2,38 & 145 & ig \\
\hline $\mathrm{Fe} / \mathrm{P}$ & 113,72 & $3.713,61$ & 395,00 & 20,77 & 146 & ig \\
\hline $\mathrm{Fe} / \mathrm{K}$ & 14,96 & 59,10 & 58,09 & 3,21 & 146 & ig \\
\hline $\mathrm{Fe} / \mathrm{Ca}$ & 6,94 & 21,92 & 45,28 & 1,57 & 146 & ig \\
\hline $\mathrm{Fe} / \mathrm{Mg}$ & 53,97 & $2.296,56$ & 540,00 & 10,24 & 146 & ig \\
\hline $\mathrm{Fe} / \mathrm{Zn}$ & 4,21 & 14,29 & 28,00 & 0,39 & 146 & ig \\
\hline $\mathrm{Fe} / \mathrm{B}$ & 2,24 & 3,65 & 11,12 & 0,27 & 146 & ig \\
\hline $\mathrm{Fe} / \mathrm{Cu}$ & 4,17 & 14,55 & 20,30 & 0,20 & 146 & ig \\
\hline $\mathrm{Fe} / \mathrm{Mn}$ & 0,40 & 0,28 & 3,41 & 0,05 & 146 & ma \\
\hline $\mathrm{Mn} / \mathrm{N}$ & 36,44 & 461,37 & 140,90 & 2,66 & 145 & ig \\
\hline $\mathrm{Mn} / \mathrm{P}$ & 510,20 & $117.972,07$ & $1.850,98$ & 32,14 & 146 & ig \\
\hline $\mathrm{Mn} / \mathrm{K}$ & 67,61 & $2.059,27$ & 290,46 & 4,41 & 146 & ig \\
\hline $\mathrm{Mn} / \mathrm{Ca}$ & 30,72 & 380,19 & 97,71 & 2,42 & 146 & ig \\
\hline $\mathrm{Mn} / \mathrm{Mg}$ & 216,59 & $15.808,85$ & 858,18 & 22,50 & 146 & ig \\
\hline $\mathrm{Mn} / \mathrm{Zn}$ & 17,99 & 335,62 & 122,22 & 1,45 & 146 & ig \\
\hline $\mathrm{Mn} / \mathrm{B}$ & 10,61 & 137,60 & 78,64 & 0,32 & 146 & ma \\
\hline $\mathrm{Mn} / \mathrm{Cu}$ & 15,41 & 213,47 & 82,76 & 0,46 & 146 & ig \\
\hline $\mathrm{Mn} / \mathrm{Fe}$ & 5,14 & 11,83 & 19,54 & 0,29 & 146 & ig \\
\hline
\end{tabular}

(1)ig, ma e me: respectivamente igual, maior e menor do que a média obtida por Pinto (2002), pelo teste t, a $1 \%$ de probabilidade.

maiores, e 3,3\% foram menores que as normas DRIS previamente publicadas. Entre as relações em que a média da relação bivariada foi maior, os nutrientes $\mathrm{Zn}$, B e Mn estavam presentes nas freqüências de $44 \%$, $33 \%$ e $33 \%$ das relações, respectivamente. Por sua vez, $\mathrm{B}$ foi o nutriente que apareceu o maior número de vezes entre as relações bivariadas com menor média $(66 \%$ das relações).
A equivalência entre os dois grupos de normas concorda com resultados obtidos na cultura da soja, em que um pequeno número de plantas sadias e de alta produtividade resultou em normas DRIS com valores equivalentes a informações derivadas de banco de dados com maior número de amostras (Hallmark et al., 1990).

A discordância verificada entre os dois grupos de normas esteve concentrada em poucos nutrientes (B, $\mathrm{Zn}$ e Mn). Essas diferenças podem ser explicadas pela amostragem, em pelo menos uma das populações, não ter refletido adequadamente as condições ambientais da região do Semi-Árido nordestino, principalmente quando são utilizados dados de parcelas experimentais de ensaios de adubação, em que um número restrito de situações nutricionais é monitorado.

A fórmula de Jones (1981) apresenta um comportamento linear entre o grau de desvio da relação nutricional a partir do valor ótimo (norma média para a relação bivariada) e o valor atribuído à respectiva função DRIS (Figura 1). Isto significa que o grau de desequilíbrio nutricional atribuído a um determinado nutriente é considerado constante nessa fórmula e dado pela inclinação da reta entre a função DRIS e a respectiva relação bivariada (Figura 1). Essa constante é definida pela magnitude estudentizada dos desvios da variável bivariada em relação à média populacional (Alvarez Venegas \& Leite, 1992).

Por meio da fórmula DRIS proposta para macronutrientes de resposta freqüente (MAF),

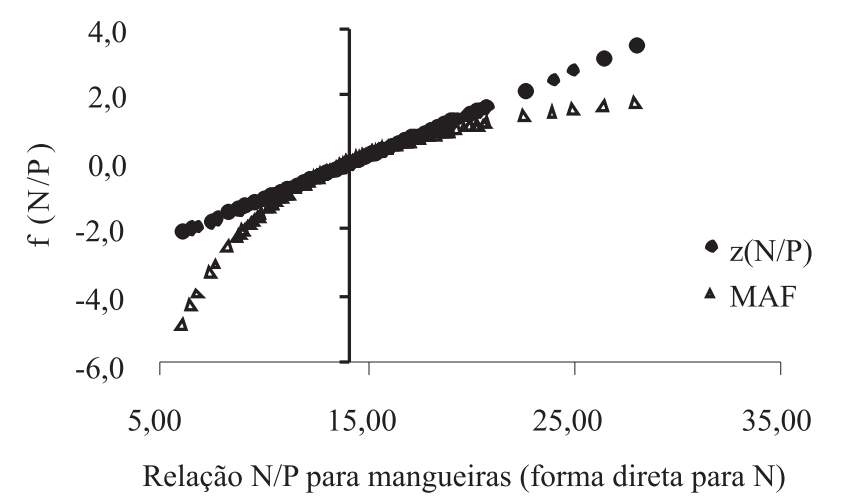

Figura 1. Valores de $\mathrm{f}(\mathrm{N} / \mathrm{P})$ em mangueiras, calculados pela fórmula de Jones (1981), z(N/P), e a fórmula para macronutrientes de resposta freqüente, quanto à relação N/P na forma direta. 
observa-se que o comportamento será distinto tanto para a forma direta da relação (Figura 1) como para a forma inversa (Figura 2), se comparado ao da fórmula de Jones (1981).

A fórmula proposta para MAF apresenta, para a função $\mathrm{f}(\mathrm{N} / \mathrm{P})$, comportamento linear em valores de N/P próximo à média da população $(\mathrm{N} / \mathrm{P} \approx \mathrm{n} / \mathrm{p})$ e, à medida que se distancia do valor médio $(\mathrm{n} / \mathrm{p})$, maior torna-se o desequilíbrio nutricional, se $\mathrm{N} / \mathrm{P}<\mathrm{n} / \mathrm{p}$, e menor será o desequilíbrio nutricional, se $\mathrm{N} / \mathrm{P}>\mathrm{n} / \mathrm{p}$, conforme Maia (1999).

Em termos de resposta biológica das plantas à disponibilidade dos macronutrientes, é possível esperar comportamentos variados entre os nutrientes avaliados. Nutrientes como N, P e K são considerados de resposta freqüente (Baldock \& Schulte, 1996) porque normalmente sua deficiência compromete a produção vegetal em maior proporção que outros nutrientes em mesmo grau de deficiência (Baldock \& Schulte, 1996; Sumner, 1999).

A fórmula original de Beaufils (1973) teria o mesmo comportamento que a fórmula proposta para MAF em situações em que $\mathrm{N} / \mathrm{P}<\mathrm{n} / \mathrm{p}$, e comportamento igual ao da fórmula de Jones (1981) para situações em que N/P>n/p. Provavelmente Beaufils (1973) previu essa situação ao introduzir o fator de correção $(\mathrm{a} / \mathrm{b}) /(\mathrm{A} / \mathrm{B})$ quando $\mathrm{A} / \mathrm{B}<\mathrm{a} / \mathrm{b}$ (Maia, 1999). Contudo, normalmente, esses mesmos nutrientes, quando em excesso, causam menor restrição à produtividade vegetal, já que podem ser acumulados nos tecidos vegetais sem causar diminuição direta da produtividade vegetal

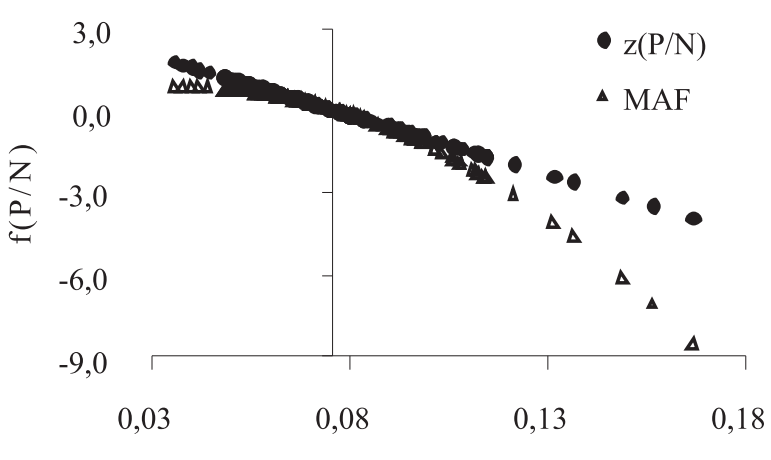

Relação P/N para mangueiras (forma inversa para N)

Figura 2. Valores de $\mathrm{f}(\mathrm{N} / \mathrm{P})$ em mangueiras, calculados pela fórmula de Jones (1981), z(N/P), e a fórmula para macronutrientes de resposta freqüente, quanto à relação N/P na forma inversa.
(Malavolta et al., 1997). Assim, a segunda correção na fórmula $(\mathrm{N} / \mathrm{P}>\mathrm{n} / \mathrm{p})$ apresenta um ajuste possível para simular o efeito do consumo de luxo sobre o equilíbrio nutricional e a produtividade das plantas.

Esse comportamento mantém-se também quando se expressa a relação N/P em sua forma inversa, ou seja, $\mathrm{P} / \mathrm{N}$ (Figura 2). Assim, no cálculo do índice DRIS para $\mathrm{N}$, por exemplo, é possível escolher a forma da relação com maior capacidade de discriminar plantas produtivas das não produtivas, mantendo-se o mesmo princípio para a correção da função DRIS para macronutrientes de resposta freqüente.

A correção proposta implica definir valores mais amplos para a função DRIS à medida que o valor da relação bivariada se afasta do valor ótimo para a relação. Ao se alterar o valor da variável $k$, utilizada como constante na maioria dos trabalhos que adotam o sistema DRIS, verifica-se que, quanto maior o valor de $\mathrm{k}$, maior o valor atribuído ao desvio nutricional pela função DRIS (Figura 3), sugerindo que essa variável seja utilizada para ajustar as funções a fim de melhorar a capacidade preditiva do estado nutricional das plantas, conforme sugerido por Maia (1999).

Essa modelagem para as funções DRIS indica que, quanto aos macronutrientes de resposta rara, a estimativa do desvio nutricional não é linear e subestima tanto a deficiência nutricional como o excesso nutricional na forma direta (Figura 4) e na forma indireta (Figura 5).

Entretanto, nos casos em que A/B é muito menor que a/b, há distorção na função DRIS, a qual tende a valores

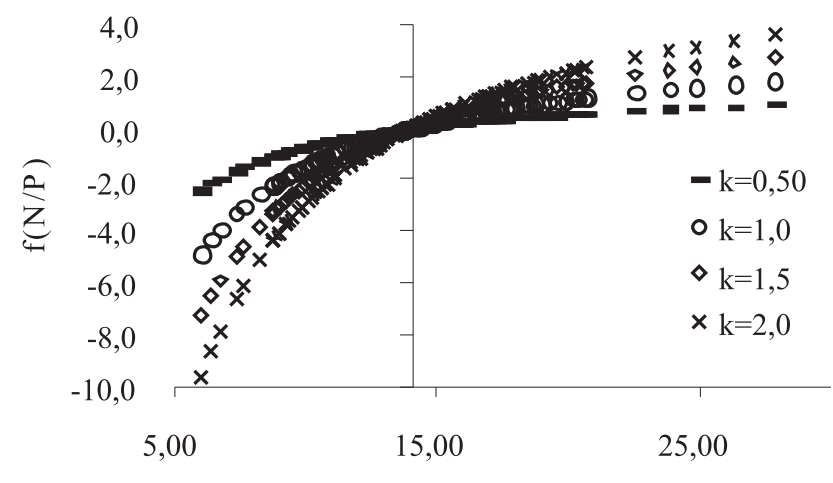

Relação N/P para mangueiras (forma direta para N)

Figura 3. Valores de f(N/P) em mangueiras, calculados pela fórmula para macronutrientes de resposta freqüente, quanto à relação N/P na forma direta, e com valores de k entre 0,5 e 2,0 . 
próximos aos do equilíbrio nutricional para relações com valores extremos de deficiência, o que é incoerente. Neste caso, é necessário o ajuste da função por meio da variável k (Figura 6), porém, também de forma limitada, o que torna o modelo indesejável. Uma alternativa consiste em não realizar correção para a condição $\mathrm{A} / \mathrm{B}<\mathrm{a} / \mathrm{b}$ e utilizar somente a variável $\mathrm{k}$ para o ajuste da função DRIS (Figura 7).

Quanto aos micronutrientes de resposta freqüente, o modelo proposto para a correção na função DRIS superestima tanto a deficiência como o excesso, e, também nesse caso, a variável $\mathrm{k}$ contribui para o ajuste do modelo, por se tratar de nutrientes com pequena

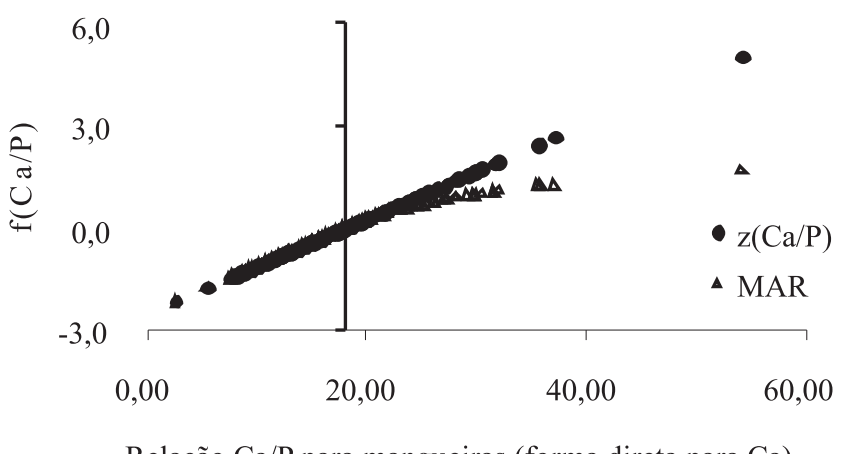

Figura 4. Valores de $\mathrm{f}(\mathrm{Ca} / \mathrm{P})$ em mangueiras, calculados pela fórmula de Jones (1981), z(Ca/P), e a fórmula para macronutrientes de resposta rara, quanto à relação $\mathrm{Ca} / \mathrm{P}$ na forma direta.

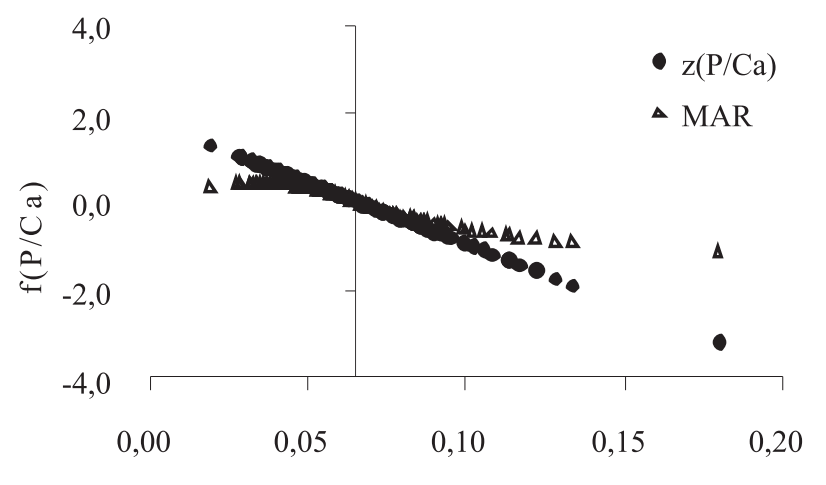

Relação P/Ca para mangueiras (forma inversa para Ca)

Figura 5. Valores de $\mathrm{f}(\mathrm{Ca} / \mathrm{P})$ em mangueiras, calculados pela fórmula de Jones (1981), z(Ca/P), e a fórmula para macronutrientes de resposta rara, quanto à relação $\mathrm{Ca} / \mathrm{P}$ na forma inversa. amplitude para valores considerados ótimos (Figura 8). Quanto aos micronutrientes de resposta rara, o modelo subestima a deficiência, porém superestima o excesso, simulando o desequilíbrio nutricional de acordo com o efeito tóxico (Figura 9). De modo semelhante às demais classes de nutrientes, a variável $\mathrm{k}$ serve para proporcionar ajustes adicionais no modelo (Figura 10).

Na fórmula para MIR, os valores possíveis para a função DRIS na relação $\mathrm{Cu} / \mathrm{P}$ são muito amplos. Essa é uma situação que ocorre com a utilização de variáveis bivariadas pouco úteis ao diagnóstico nutricional e, nestes casos, a adoção de critérios estatísticos para identificar as relações a serem utilizadas no cálculo dos índices

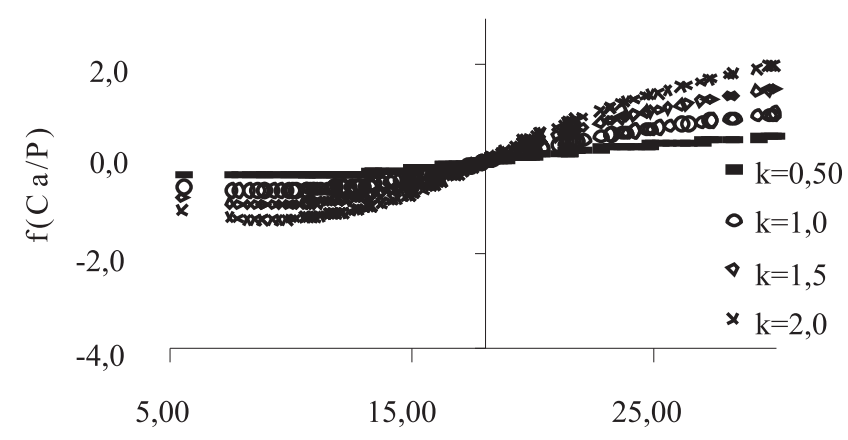

Relação $\mathrm{Ca} / \mathrm{P}$ para mangueiras (forma direta para $\mathrm{Ca}$ )

Figura 6. Valores de $\mathrm{f}(\mathrm{Ca} / \mathrm{P})$ em mangueiras, calculados pela fórmula para macronutrientes de resposta rara, quanto à relação $\mathrm{Ca} / \mathrm{P}$ na forma direta, e com valores de $\mathrm{k}$ entre 0,5 e 2,0.

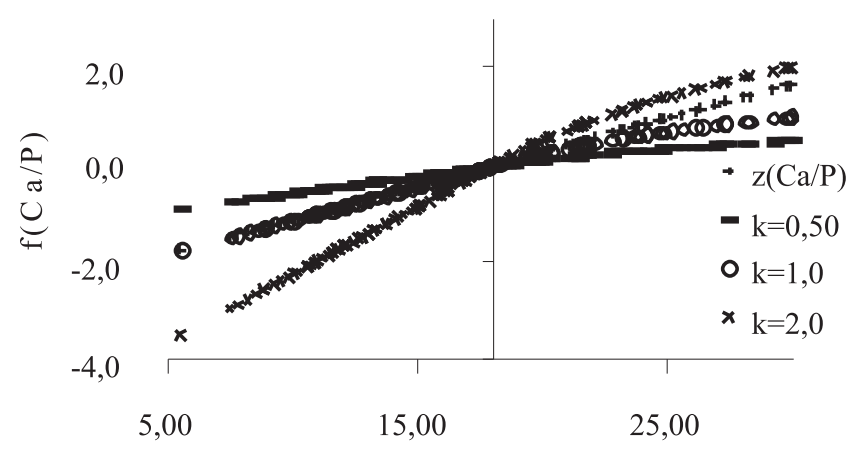

Relação $\mathrm{Ca} / \mathrm{P}$ para mangueiras (forma direta para $\mathrm{Ca}$ )

Figura 7. Valores de $\mathrm{f}(\mathrm{Ca} / \mathrm{P})$ em mangueiras, calculados pela fórmula para macronutrientes de resposta rara sem correção para $\mathrm{A} / \mathrm{B}<\mathrm{a} / \mathrm{b}$, quanto à relação $\mathrm{Ca} / \mathrm{P}$ na forma direta, e com valores de k entre 0,5 e 2,0 . 


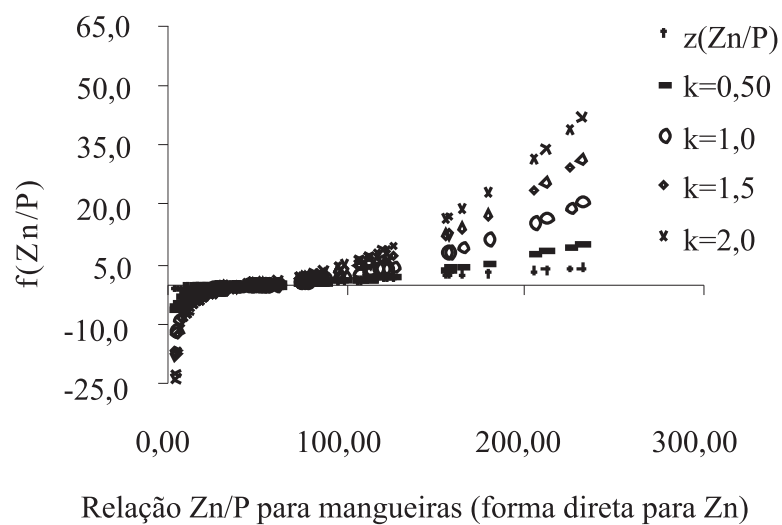

Figura 8. Valores de $\mathrm{f}(\mathrm{Zn} / \mathrm{P})$ em mangueiras, calculados pela fórmula para micronutrientes de resposta freqüente, quanto à relação Zn/P na forma direta, e com valores de k entre 0,5 e 2,0.

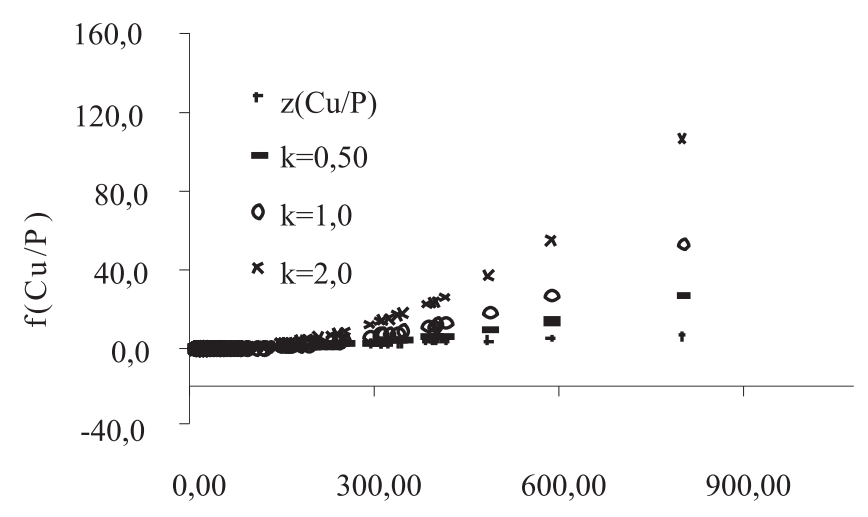

Relação $\mathrm{Cu} / \mathrm{P}$ para mangueiras (forma direta para $\mathrm{Cu}$ )

Figura 9. Valores de $\mathrm{f}(\mathrm{Cu} / \mathrm{P})$ em mangueiras, calculados pela fórmula para micronutrientes de resposta rara, quanto à relação $\mathrm{Cu} / \mathrm{P}$ na forma direta, e com valores de $\mathrm{k}$ entre 0,5 e 2,0.

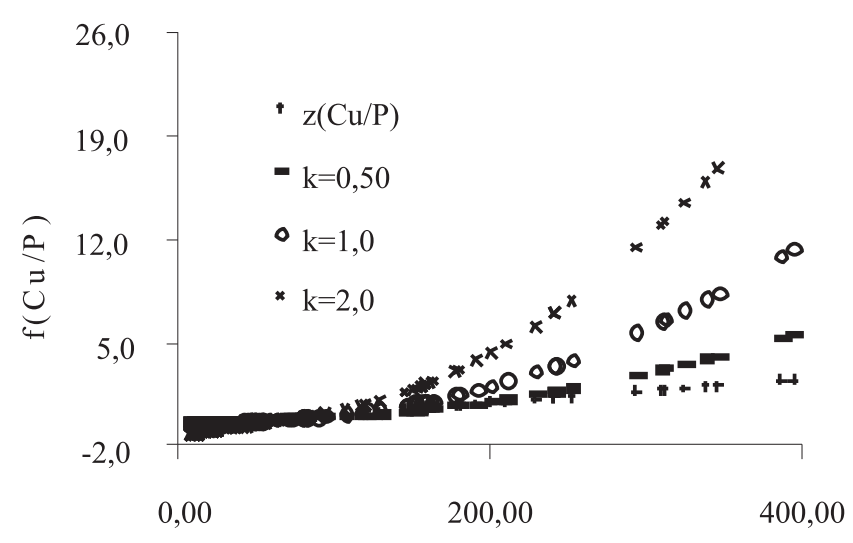

Relação $\mathrm{Cu} / \mathrm{P}$ para mangueiras (forma direta para $\mathrm{Cu}$ )

Figura 10. Valores de $\mathrm{f}(\mathrm{Cu} / \mathrm{P})$ em mangueiras, calculados pela fórmula para micronutrientes de resposta rara, quanto à relação $\mathrm{Cu} / \mathrm{P}$ na forma inversa, com valores de $\mathrm{k}$ entre 0,5 e 2,0.
DRIS resulta em melhor concordância entre o valor, o equilíbrio nutricional e a produtividade vegetal (Wadt et al., 1999).

A utilização de muitos nutrientes no método DRIS tem diminuído a precisão do diagnóstico produzido, o que resulta em diferentes tentativas de melhorar as fórmulas de cálculo dos índices DRIS (Wadt, 1996). Contudo, o uso de diferentes fórmulas para as funções DRIS, aliado ao uso de valores diferenciados para a variável k, cria comportamentos distintos para as funções DRIS mais fáceis de serem ajustados ao índice de desequilíbrio nutricional esperado para cada tipo de nutriente.

\section{Conclusão}

A modelagem das funções DRIS e da variável k permite ajustes nas fórmulas DRIS capazes de superestimar ou subestimar os valores dos desvios nutricionais para situações de deficiência e de excesso de nutrientes.

\section{Agradecimentos}

À Embrapa, pelos recursos financeiros.

\section{Referências}

ALVAREZ VENEGAS, V.H.; LEITE, R.A. Fundamentos estatísticos das fórmulas para cálculo dos índices dos nutrientes no sistema integrado de diagnose e recomendação (DRIS). In: REUNIÃO BRASILEIRA DE FERTILIDADE DO SOLO E NUTRIÇÃO DE PLANTAS, 20., 1992, Piracicaba. Anais. Piracicaba: SBCS, 1992. p.186-188.

BALDOCK, J.O.; SCHULTE, E.E. Plant analysis with standardized scores combines DRIS and sufficiency range approaches for corn. Agronomy Journal, v.88, p.448-456, 1996.

BATAGLIA, O.C.; QUAGGIO, J.A.; SANTOS, W.R. dos; ABREU, M.F. de. Diagnose nutricional do cafeeiro pelo DRIS variando-se a constante de sensibilidade dos nutrientes de acordo com a intensidade e frequiência de resposta na produção. Bragantia, v.63, p.253-263, 2004.

BATAGLIA, O.C.; SANTOS, W.R. Efeito do procedimento de cálculo e da população de referência nos índices do sistema integrado de diagnose e recomendação (DRIS). Revista Brasileira de Ciência do Solo, v.14, p.339-344, 1990.

BEAUFILS, E.R. Diagnosis and recommendation integrated system (DRIS): a general scheme of experimentation and calibration based on principles developed from research in plant nutrition. Pietermaritzburg: University of Natal, 1973. 132p.

ELWALI, A.M.O.; GASCHO, G.J. Soil testing, foliar analysis, and DRIS as guide for sugarcane fertilization. Agronomy Journal, v.76, p.466-470, 1984. 
JONES, C.A. Proposed modifications of the diagnosis and recommendation integrated system (DRIS) for interpreting plant analysis. Communications in Soil Science and Plant Analysis, v.12, p.785-794, 1981.

HALLMARK, W.B.; BEVERLY, R.B.; PARKER, M.B.; ADAMS, J.F.; BOSWELL, F.C.; OHKI, K.; SHUMAN, L.M.; WILSON, D.O. Evaluation of soybeans zinc and manganese requirements by the M-DRIS and sufficiency range methods. Agronomy Journal, v.81, p.770-776, 1989.

HALLMARK, W.B.; BEVERLY, R.B.; SUMNER, M.E.; MOOY, C.J. de; MORRIS, H.F.; PESEK, J.; FONTENOT, J.D. Soybean phosphorus and potassium requirement evaluation by three M-DRIS data bases. Agronomy Journal, v.82, p.323-328, 1990.

HALLMARK, W.B.; WALWORTH, J.L.; SUMNER, M.E.; MOOY, C.J. de; PESEK, J.; SHAO, K.P. Separating limiting from non-limiting nutrients. Journal of Plant Nutrition, v.10, p.1381-1390, 1987.

MAIA, C. Análise crítica da fórmula original de Beaufils no cálculo dos índices DRIS: a constante de sensibilidade. In: WADT, P.G.S.; MALAVOLTA, E. (Org.). Monitoramento nutricional para a recomendação de adubação de culturas. Piracicaba: Potafos, 1999. 1 CD-ROM.

MALAVOLTA, E.; VITTI, G.C.; OLIVEIRA, S.A. de. Avaliação do estado nutricional de plantas: princípios e aplicação. 2.ed., rev. e atual. Piracicaba: Potafos, 1997. 319p.

NICK, J.A. DRIS para cafeeiros podados. 1998. 86p. Dissertação (Mestrado) - Escola Superior de Agricultura Luiz de Queiroz, Piracicaba.

PARENT, L.E.; ISFAN, D.; TREMBLAY, N.; KARAM,A. Multivariate nutrient diagnosis of the carrot crop. Journal of the American Society for Horticultural Science, v.119, p.420-426, 1994.

PARTELLI, F.L.; VIEIRA, H.D.; COSTA, A.N. da. Diagnóstico nutricional em cafeeiro conilon orgânico e convencional no Espírito Santo, utilizando o DRIS. Ciência Rural, v.35, p.1456-1460, 2005.

PINTO, P.A. da C. Avaliação do estado nutricional da mangueira Tommy Atkins pelo DRIS e da qualidade pós-colheita de frutos no Submédio São Francisco. 2002. 124p. Dissertação (Doutorado) - Universidade Federal de Viçosa, Viçosa.
SCHAFFER, B.; LARSON, K.D.; SNYDER, G.H.; SANCHEZ, C.A. Identification of mineral deficiencies associated with mango decline by DRIS. HortScience, v.23, p.617-619, 1988.

SILVA, D.J.; QUAGGIO, J.A.; PINTO, P.A. da C.; PINTO, A.C.Q. de; MAGALHÃES, A.F. de J. Nutrição e adubação. In: GENÚ, P.J. de C.; PINTO, A.C.Q. de (Ed.). A cultura da mangueira. Brasília: Embrapa Informação Tecnológica, 2002. p.191-221.

SILVA, G.G.C. da; NEVES, J.C.L.; ALVAREZ VENEGAS, V.H.; LEITE, F.P. Avaliação da universalidade das normas DRIS, M-DRIS e CND. Revista Brasileira de Ciência do Solo, v.29, p.755-761, 2005.

SILVEIRA, C.P.; NACHTIGALL, G.R.; MONTEIRO, F.A. Testing and validation of methods for the diagnosis and recommendation integrated system for Signal grass. Scientia Agricola, v.62, p.520$527,2005$.

SUMNER, M.E. Effect of corn leaf sampled on N, P, K, Ca and Mg content and calculated DRIS indexes. Communications in Soil Science and Plant Analysis, v.8, p.269-280, 1977b.

SUMNER, M.E. Preliminary N, P, and K foliar diagnostic norms for soybeans. Agronomy Journal, v.69, p.226-230, 1977a.

SUMNER, M.E. The use and misuse of the diagnosis and recommendation integrated system (DRIS) in foliar diagnosis. In: WADT, P.G.S.; MALAVOLTA, E. (Org.). Monitoramento nutricional para a recomendação de adubação de culturas. Piracicaba: Potafos, 1999. 1 CD-ROM.

WADT, P.G.S. Nutritional status of Eucalyptus grandis clones evaluated by critical level and DRIS methods. Revista Árvore, v.28, p.15-20, 2004.

WADT, P.G.S. Os métodos da chance matemática e do sistema integrado de diagnose e recomendação (DRIS) na avaliação nutricional de plantios de eucalipto. 1996. 99p. Dissertação (Doutorado) - Universidade Federal de Viçosa, Viçosa.

WADT, P.G.S.; NOVAIS, R.F. de; ALVAREZ VENEGAS, V.H.; BRAGANÇA, S.M. Alternativas da aplicação do DRIS à cultura de café Conilon (Coffea canephora Pierre). Scientia Agricola, v.56, p.83-92, 1999.

Recebido em 26 de abril de 2006 e aprovado em 9 de outubro de 2006 